\title{
Evaluation of the capacity of the DNA barcode ITS2 for identifying and discriminating dryland plants
}

\author{
W.S. Al-Juhani \\ Makkah, KSA \\ Corresponding author: W.S. Al-Juhani \\ E-mail: wsjuhani@uqu.edu.sa \\ Genet. Mol. Res. 18 (1): gmr18133 \\ Received September 02, 2018 \\ Accepted February 21, 2019 \\ Published March 28, 2019 \\ DOI http://dx.doi.org/10.4238/gmr18133
}

Biology Department, Faculty of Applied Science, Umm Al-Qura University,

\begin{abstract}
Many of the plants that grow in arid and semi-arid regions under harsh conditions (drought, salinity and misuse) are of medical importance. Desert plants also provide other benefits; so conservation efforts should be strengthened in these areas. In this study, three DNA barcodes - chloroplast maturase-K (matK), ribulose-1,5-bisphosphate carboxylase/oxygenase (rbcL) and secondary internal transcribed spacer (ITS2) - were tested in order to determine the ability of these markers to distinguish between various species. A total of 93 fresh and dry samples belonging to 30 plant families were examined. These are native to the arid lands around Makkah in the Kingdom of Saudi Arabia. Various tools and tests were used to evaluate the efficiency of the barcode markers; these included the basic local alignment search tool (BLAST), as well as the use of inter- and intraspecific distances, barcode gap, prediction of the secondary structure of the ITS2 and phylogenetic methods. The results confirmed the efficiency and superiority of the ITS2 loci over two chloroplast genes: matK and rbcL. ITS2 had a 97\% success rate with amplification and a 95\% success rate with sequencing, showing $100 \%$ identification at the family level, $77 \%$ identification at the genus level and $70 \%$ species resolution. A clear barcode gap was observed between inter- and intraspecific distances. Identification was achieved even in cases of degraded samples or old herbarium
\end{abstract}


specimens. This study contributes to DNA barcode data by adding 224 DNA sequences of desert plants to the National Center for Biotechnology Information NCBI database. The results of this study are important and useful for application in each of the following fields: validation of medicinal plant identity, conservation, and taxonomic and evolutionary studies.

Key words: DNA; Barcodes; ITS2; rbcL; matK; Desert plant

\section{INTRODUCTION}

Identification of organisms has important applications. However, determining a species based on phenotypic traits is a difficult and time-consuming task that requires expert input, which is not always available. DNA barcoding is emerging as a reliable method for species identification. It uses a short sequence of a universal standard part of a genome instead of using the whole genome in order to verify the identity of specimens.

The Mitochondrial CO1 region has been recommended as the standard barcode for animals (Hebert et al., 2003). However, the mitochondrial genome is not suitable for DNA barcodes in land plants because it slowly evolves with a low rate of substitution (Fazekas et al., 2008). This affects the use of this genome to distinguish between plant species. The chloroplast genome is used as the standard in plants because it has a stable genetic structure in haploid form and is parentally inherited (Xiwen et al., 2015).

The Consortium for the Barcode of Life ([CBOL]; CBOL Plant Working Group, 2009) recommended the use of chloroplast maturase-K (matK) and ribulose-1,5bisphosphate carboxylase/oxygenase ( $\mathrm{rbcL}$ ) genes as a universal barcode for vascular

plants. There has been a clear increase in DNA barcode applications in recent years, for example, for resolving identification and taxonomy issues. Different types of DNA barcodes have been applied to plant species, but the most frequently used are matK and rbcL markers. These were used for identification and taxonomic studies of five species of the genus Andrographis, belonging to the family Acanthaceae (Arolla et al., 2015). Lin et al. (2015) used 27 prescreened chloroplast DNA (cpDNA) regions to screen Phalaenopsis species. Similarly, Nithaniyal and Parani (2016) used rbcL, matK, internal transcribed spacer 2 (ITS2) and trnH-psbA markers for identification of Terminalia spp. Martin et al. (2016) reported that DNA barcoding of ITS in the nuclear ribosomal DNA (nrDNA) region supported the separation of Chroogomphus mediterraneus from C. rutilus. In Mezzasalma et al.'s (2017) study, four barcode loci - rbcL, matK, trnH-psbA and ITS - were used to identify the traditional medicine species Cyperus articulatus within the natural flora of the Amazon.

This method is also used to check herbal medicines. As well as using DNA barcodes for phylogenetic analysis and genetic diversity, Patil et al. (2016) used eight plastid (matK, rbcL, trnH-psbA, rpoC1, ycf5, rpoB, atp and psbK-psbI) and six nuclear ITS markers to examine the phylogenetic relationships among 19 species of the genus Chlorophytum. Silva et al. (2015) studied the phylogenetic relationships among 18 species of Spondias of the family Anacardiaceae using cpDNA barcodes for the trnH-psbA spacer gene. In addition to using the chloroplast genome for detecting aquatic invasive plant species, Scriver et al. (2015) examined three regions - matK, rbcL and trnH-psbA in 10 
species of invasive plants. Yang et al. (2017) examined intraspecific differences among 13 species in the subgenus Potamogeton in China using seven cpDNA barcodes (trnL-trnLtrnF, rpl20-rps12, trnG intron, trnH-psbA, matK and $\mathrm{rbcL}$ ) and two nuclear markers (ITS and PHYB), They recommended used PHYB as the most efficient candidate to detect divergence among Potamogeton species.

Although the chloroplast genes matK and rbcL have become the most commonly used loci in land plants, several studies have identified problems with these two markers. The rbcL gene has usually been successfully amplified and has given good sequencing results, but low variation in sequence data affects its discriminatory power (Michel et al., 2016; Mohamed, 2016). With regard to matK, amplification and application has been difficult in many families, and it cannot be considered a universal barcode region. Standard DNA barcoding must be present in most taxa levels; with easy amplification and sequencing, and they should have wide variation across species and little variation within a species (Sarvananda, 2018).

Several researchers (Pan et al., 2009; Chen et al., 2010; Braukmann et al., 2017) have shown the ability of ITS2 to discriminate between specimens of different genera. The suitability of the ITS2 region for use as a DNA barcode was tested by Chen et al. (2010) on 6,600 specimens, representing 4,800 species and 753 genera of the most important medicinal plants in China. They compared its power of discrimination to that of six other loci (trnH-psbA, matK, rbcL, rpoC1, ycf5 and ITS). The results confirmed that ITS2 had superiority over the rest of the markers, with a $92.7 \%$ rate of successful identification. Most recently, a study by Braukmann et al. (2017) involved a large number of plants and families and covered a wide range of geographical diversity. They used three DNA barcode regions (rbcL, matK and ITS2) to identify 4,923 species, 1,153 genera and 171 families of vascular plants that grow in Canada. The results showed that rbcL had the highest percentage of amplification and sequencing (90-100\%), followed by ITS2 ( 90\%), whereas matK had the lowest percentage $(56-90 \%)$. ITS2 had the highest level of discriminating power and species resolution (79-93\%), followed by matK (45-80\%) and rbcL (17\%-92\%). Yu et al. (2017) found that even with a short sequence length (ranging from 220 to $241 \mathrm{bp}$ ), ITS2 was $100 \%$ successful in identifying 127 samples of Trachelospermum jasminoides used in traditional Chinese medicine.

The Kingdom of Saudi Arabia (KSA) is located in Southwest Asia. The Rub AlKhali desert covers the southern areas of the kingdom's topography; it is the largest continuous sand desert in the world (Vincent, 2008). The climate of the KSA is dry and hot in summer, with an average temperature of around $35^{\circ} \mathrm{C}$, but temperatures can reach $50^{\circ} \mathrm{C}$ and above between June and August. Many of the plants in arid environments have medical importance and are still used in traditional medicine (Sher and Aldosari, 2012; AL-Eisawi and Al-Ruzayza, 2015). Reliable methods for identifying these plants are critical not only because of the medical value of these plants but also because of the need to preserve the natural diversity of flora in this desert environment.

Several previous studies have applied DNA barcoding techniques to plants native to the KSA. Bafeel et al. (2012c) used matK and rbcL to identify Chenopodium murale (Chenopodiaceae), and Bafeel et al. (2012b) used the rbcL gene to identify Coptosperma graveolens subsp. arabicum (Rubiaceae). Khan et al. (2012a) used nuclear DNA ITS and chloroplast rpoB and rpoC1 markers to identify Ochradenus arabicus (Resedaceae). Bafeel et al. (2012a) used rbcL for identification and conservation of the endemic tree Mimusops 
laurifolia (Sapotaceae). Al-Qurainy et al. (2013) used the nrDNA region ITS1-5.8S-IT5 to assess the relationships among five rare species in the KSA: Scadoxus multiflorus (Acanthaceae), Pancratium tortuosum (Amaryllidaceae), Senecio hadiensis (Asteraceae), Commiphora myrrha (Burseraceae) and Heliotropium strigosum (Boraginaceae). Alaklabi et al. (2014) used the rbcL gene to identify the endangered tree Diospyros mespiliformis (Ebenaceae). Furthermore, Al-Qurainy et al. (2014) used the nuclear gene ITS for identification of Nepeta deflersiana (Lamiaceae). Ali et al. (2015) used ITS1 and ITS4 primers in DNA barcodes to study the system of the genus Echinops (Asteraceae). Khan et al. (2017) used six DNA barcodes - matK, rbcL, rpoB, rpoC1, atpF-atpH and psbK - to identify the dryland medicinal plant Rhazya stricta (Apocynaceae).

Comparisons among these results are difficult because of variations in the types of DNA barcode markers used. Most of these studies focused on a particular family or genus, except that of Bafeel et al. (2011), which compared dryland plant samples and evaluated the universal primers matK and rbcL located in 26 plant species from 14 plant families native to Saudi Arabia. This study recorded a higher success rate in amplification with rbcL (88\%) than with matK (69\%), though both failed to amplify the DNA of three species of the family Asteraceae. The PCR products of matK were less than the expected size for two species: Malva parviflora (Malvaceae) and Salsola imbricate (Chenopodiaceae). Bafeel et al. (2011) recommended the use of a further set of primers that can be applied to a wide range of plant species.

Our work had the following aims: 1) to examine and compare the possible use of the molecular DNA barcodes ITS2, matK and rbcL for identifying desert plants; 2) to determine the best of these three DNA barcode markers for resolving identification questions and for discriminating among native KSA flora; and 3) to apply the recommended universal barcodes to plants from different geographic regions and conditions.

\section{MATERIAL AND METHODS}

\section{Study Area}

The samples were collected from the Makkah district in Western KSA. The plants in this region are adapted to growth in harsh conditions, including high temperatures, drought and salinity, all of which impact the population. The number and types of plants recorded in this area vary due to variable amounts of rain. Meelad (1987) recorded nearly 70 species native to the geographical area of Makkah, while Abdel et al. (2013) reported 104 species growing in the Makkah region.

\section{Plant Material}

The study samples included fresh leaves collected from 40 specimens from the Makkah region in 2016 - 2017, which were dried in silica gel. An additional 53 herbarium specimens were obtained from the Biology Department of Umm Al-Qura University (Makkah), which had been collected from 1991 to 2015 from various locations in Makkah. The total number of samples was 93, with 30 families, 71 genera and 80 species. The samples comprised Makkah's most important native flora, representing more than $70 \%$ of the genera recorded in the region by Meelad (1987) and Abdel et al. (2013). (All 
information relating to the study samples is available in Table S1.) The plants were identified based on their morphology by referring to various sources (Migahid, 1996; Chaudhary, 2000, 2001; e-floras.org: http://www.efloras.org) and were assigned to their respective families.

\section{DNA Extraction}

Samples of dry leaves were collected from each plant. Total genomic DNA was isolated from the leaves using a magnetic bead-based DNA extraction method (Azimi et al., 2011). The quantity of extracted DNA was estimated using a Qubit 3.0 Fluorometer (Thermo-scientific, Wilmington, USA). The quality of the DNA was checked using $1 \%$ agarose gel electrophoresis and visualized under ultraviolet light, and the isolated DNA was stored at $-20^{\circ} \mathrm{C}$.

\section{Primer Sequences}

The DNA regions rbcL and matK were amplified with universal DNA barcoding primers (Plant Working Group, 2009). For the ITS2 locus, the primer sequences published by Chen et al. (2010) were used. Table 1 shows the primers' forward and reverse sequences for each locus.

Table 1. Primers used for amplification of ITS2, matk and rbcl barcodes.

\begin{tabular}{|c|c|}
\hline Region & Primers Sequences F/R \\
\hline \multirow{2}{*}{ ITS2 } & 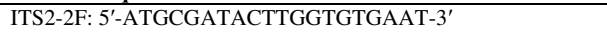 \\
\hline & ITS2-3R: 5'-GACGCTTCTCCAGACTACAAT-3' \\
\hline \multirow{2}{*}{ matK } & matK-KIM3F: 5'-CGTACAGTACTTTTGTGTTTACGAG3' \\
\hline & matK-KIM1R: 5' ACCCAGTCCATCTGGAAATCTTGGTTC-3' \\
\hline \multirow{2}{*}{$\mathrm{rbcL}$} & rbcLa-F: 5'-ATGTCACCACAAACAGAGACTAAAGC-3' \\
\hline & rbcLa-R: 5'-GTAAAATCAAGTCCACCRCG-3' \\
\hline
\end{tabular}

\section{DNA Amplification and Sequencing}

PCRs of rbcL and matK markers for the chloroplast genome were amplified following the method described by Maloukh et al. (2017), using a total volume of $50 \mu \mathrm{L}$, including $25 \mu \mathrm{L}$ of Taq PCR Master Mix, $22 \mu \mathrm{L}$ of distilled water, $1 \mu \mathrm{L}$ of forward primer, $1 \mu \mathrm{L}$ of reverse primer and $1 \mu \mathrm{L}$ of the DNA template $(50-80 \mathrm{ng} / \mu \mathrm{L})$. Amplification was performed in a Veriti 96-Well Fast Thermal Cycler (Applied Biosystems, CA, USA), with an initial denaturation at $94^{\circ} \mathrm{C}$ for $3 \mathrm{~min}$. This was followed by 35 cycles at $94^{\circ} \mathrm{C}$ for $1 \mathrm{~min}$ and an annealing temperature at $55^{\circ} \mathrm{C}$ for $1 \mathrm{~min}$, followed by $72^{\circ} \mathrm{C}$ for $1 \mathrm{~min}$ and a final elongation at $72^{\circ} \mathrm{C}$ for $7 \mathrm{~min}$.

The ITS2 region was amplified following the method described by Chen et al. (2010), using $25-\mu \mathrm{L}$ reaction volumes containing $12.5 \mu \mathrm{L}$ of Master Mix, $8.5 \mu \mathrm{L}$ of molecular grade water, $1 \mu \mathrm{L}$ of each primer and $2 \mu \mathrm{L}$ of the DNA template. Thermal cycling was conducted with an initial denaturation at $94^{\circ} \mathrm{C}$ for $5 \mathrm{~min}$, followed by 40 cycles at $94^{\circ} \mathrm{C}$ for $45 \mathrm{~s}, 56^{\circ} \mathrm{C}$ for $45 \mathrm{~s}, 72^{\circ} \mathrm{C}$ for $1.5 \mathrm{~min}$ and a final extension final step at $72^{\circ} \mathrm{C}$ for 10 min. The successful amplifications of the ITS2, matK, and rbcL PCR products were 
checked by electrophoresis on a 1.5\% agarose gel and by imaging using a UVP system; sequencing was performed at Laragen Inc. (Culver City, CA, USA).

\section{Bioinformatics Data Analysis}

Forward and reverse sequences were assembled into contig sequences per sample and per gene, performed in DNA Baser v. 4.16.0 (2013). The ITS2 region in each specimen was detected using a hidden Markov model to remove the $5.8 \mathrm{~S}$ and $28 \mathrm{~S}$ sections, which may overlap with the ITS2 region (Keller et al., 2009). A multiple sequence alignment was run for each gene using the Muscle algorithm in MEGA5.2 (Tamura et al., 2011). The sequences were manually adjusted, and ambiguous regions were removed.

The species resolution of the three DNA barcodes - ITS2, matK and rbcL - was assessed using the basic local alignment search tool (BLAST) and the following methods: pairwise genetic distance, inter- and intraspecific distance, barcode gap, prediction of the secondary structure of the ITS2 and phylogenetic methods.

The BLAST was run against known specimens in GenBank. The identification process took place on three levels: family, genus and species. The query sequences were detected by selecting the highest maximum score and lowest E-value (Gao et al., 2011). Outcomes were classified into three levels: A - correct identification, meaning determination of the right species, genus or family; B - ambiguous identification, meaning determination of several species, genera or families that included the right one; and $\mathrm{C}-$ incorrect identification, meaning determination of species, genera or families that were not correct (Meier et al., 2006).

Pairwise genetic distances for the ITS2, matK and rbcL regions in the three levels were calculated using the Kimura two-parameter (K2P) model in MEGA 7.0.27 software (Tamura et al., 2011; Kumar et al., 2016). Inter- and intraspecific distances were calculated for the three barcode genes using the SpeciesIdentifier window in TaxonDNA v.1.7.8 (Meier et al., 2006). Barcode gaps in matK, rbcL and ITS2 were analysed using the Automatic Barcode Gap Discovery (ABGD) (Puillandre et al., 2012) based on K2P distance.

The secondary structure of the ITS2 sequences was predicted using the ITS2 ribosomal RNA database (http://its2.bioapps.biozentrum.uni-wuerzburg.de/; Schultz et al., 2005). The trees of phylogenetic relationships were obtained by applying the neighbourjoining (NJ) method, using the K2P model and 1000 bootstrap replications in MEGA 7.0.27 software (Kumar et al., 2016). The tree of each gene was evaluated based on the ability of the species under each family to cluster in one clade or to form a monophyletic group, in addition to a bootstrap supporting value.

\section{RESULTS}

\section{PCR and Sequencing}

Genomic DNA isolation was successful for all the samples. PCR amplifications of 93 plant specimens yielded 90 results for rbcL (97\%), 82 for matK $(88 \%)$ and 90 for ITS2 (97\%), as shown in Table 2. Ninety (97\%), 82 (88\%) and 88 (95\%) of the specimens were 
successfully sequenced in the rbcL, matK and ITS2 loci, respectively. The sequence length ranged from 201 to $348 \mathrm{bp}$ for ITS2, 792 to $964 \mathrm{bp}$ for matK and 584 to $628 \mathrm{bp}$ for rbcL.

Sequencing success varied according to the family. The lowest success rates were found for matK in members of Ephedraceae (50\%) and Poaceae $(12.5 \%)$ families. There was a mixed sequence in ITS2 for the Apocynaceae family (20\%). However, RbcL, matK and ITS2 gave $100 \%$ amplification and sequencing success in some families, such as Amaranthaceae, Asteraceae and Fabaceae.

In terms of sequence length for ITS2, the longest sequence was from Forsskaolea tenacissima (Urticaceae; $348 \mathrm{bp}$ ), and the shortest was from Farsetia aegyptia (Brassicaceae; 201 bp). The longest matK sequence was from Malva parviflora (Malvaceae) with $964 \mathrm{bp}$, while the shortest was $792 \mathrm{bp}$, which was produced by Panicum turgidum (Poaceae). Of the rbcL sequences, Boerhavia diffusa (Nyctaginaceae) was the longest (628 bp), while the shortest at 584 bp was Cotula abyssinica (Asteraceae; see Table 2).

The guanine-cytosine content (GC) ranged from 40.6 to 66.5 with an average of 57.1 in ITS2, whereas in matK, the range was $27.8-37.8$ with an average of 33.2. In rbcL, the GC percentage ranged from 40.6 to 58.4 (43.3 average). These results are shown in Table 2.

Table 2. Evaluation of three DNA barcoding regions used in the study of dryland plants.

\begin{tabular}{llll}
\hline \multirow{2}{*}{ Variable } & \multicolumn{2}{c}{ DNA region } \\
\cline { 2 - 4 } & ITS2 & matK & rbcL \\
\hline Number of samples & 93 & 93 & 93 \\
Mean and range of GC content (\%) & $57.1(40.6-66.5)$ & $33.2(27.8-37.8)$ & $43.3(40.6-58.4)$ \\
Efficiency of PCR amplification (\%) & $90(97 \%)$ & $82(88 \%)$ & $90(97 \%)$ \\
Success rate of sequencing (\%) & $88(95 \%)$ & $82(88 \%)$ & $90(97 \%)$ \\
Amplified product length (bp) & $\sim 200$ & $\sim 800$ & $\sim 600$ \\
Mean and range of sequenced length (bp) & $227(201-348)$ & $870.33(792-964)$ & $601(584-628)$ \\
\hline
\end{tabular}

\section{Species Resolution and Barcode Analysis}

Based on the BLAST data, the sequence homology had a mean of 98, 95 and $99 \%$ for ITS2, matK and rbcL, respectively. The mean of the inquired sequences was 86, 95 and $98 \%$ for ITS2, matK and rbcL, respectively. E-values were $\leq(0)$ for the three genes.

ITS2 sequences were correctly identified by $100,77.2$ and $70.4 \%$ at family, genus and species levels, respectively. In terms of ambiguous sequences, identification was 4.6 and $6.8 \%$ for genus and species levels, while ITS2 identification was incorrect at 18.1 and $22.7 \%$ for the genus and species levels, respectively (see Figure 1). For the matK sequences, the specimens were correctly assigned by 100, 66.6 and 53.9\% at family, genus and species levels, whereas ambiguous detection was 11.1 and $17.7 \%$ for genus and species levels, respectively. MatK identification was incorrect by 22.2 and $28.3 \%$ for genus and species levels, respectively. The rbcL successfully assigned sequences at the levels of family $(100 \%)$, genus $(63.4 \%)$ and species $(45.5 \%)$, whereas identification was ambiguous by 1.2 and $22.2 \%$ for genus and species levels, respectively. Incorrect rbcL identification was observed for genus (35.3\%) and species (32.2\%) levels, as shown in Figure 1. 


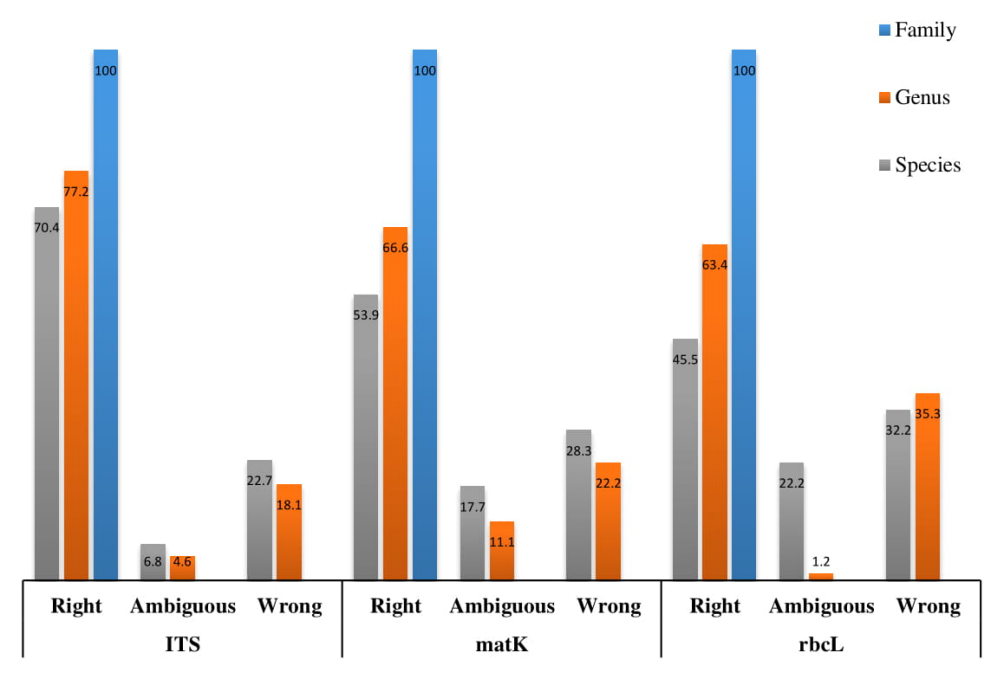

Figure 1. Identification success of dryland plant specimens at three levels (family, genus and species) using BLAST and matching sequences to the NBCI database. Total sequences for rbcL $=90$, ITS2 $=88$ and matK $=82$. The values in the columns were estimated in percentages $(\%)$.

Interestingly, the ITS2 sequences for Phyllanthus rotundifolius, Dipterygium glaucum, Steinheilia radicans, Tetraena coccinea, Tetraena simplex and Tripteris vaillantii were not available in either the NCBI or Barcode of Life databases. The following matK groups were also unavailable in the GenBank database: Phyllanthus rotundifolius, Leptadenia pyrotechnica, Forsskaolea tenacissima, Tephrosia uniflora, Heliotropium europaeum L., Heliotropium strigosum and Cotula abyssinica.

The interspecific distances for the three barcode loci using TaxonDNA software are shown in Table 3. The ITS2 locus gave a higher interspecific mean than the two other markers $(0.76 \%)$; rbcL gave the lowest value $(0.38 \%)$, while the matK value was $0.63 \%$. The intraspecific distances were the lowest in ITS2 (0.03) and were 0.08 and 0.10 for rbcL and matK, respectively, which reflects the utility of the ITS2 marker.

Table 3. Inter- and intraspecific distance for the three barcode loci and dryland plant samples using the taxon DNA software.

\begin{tabular}{lllllll}
\hline \multirow{2}{*}{ Locus } & \multicolumn{3}{l}{ Intraspecific distance } & \multicolumn{3}{l}{ Interspecific distance } \\
\cline { 2 - 6 } & Min. & Max. & Mean. & Min. & Max. & Mean. \\
\hline ITS2 & 0.00 & 0.49 & 0.03 & 0.00 & 0.82 & 0.76 \\
matK & 0.00 & 0.73 & 0.10 & 0.00 & 0.76 & 0.63 \\
rbcL & 0.00 & 0.75 & 0.08 & 0.00 & 0.79 & 0.38 \\
\hline
\end{tabular}

The comparison between specimens in terms of the pairwise genetic distance presented in Table 4, show that the mean genetic distance of the ITS2 locus was much higher than the mean distance of both marK and rbcL loci at three taxa levels for the three genes. 
Table 4. Genetic divergence of dryland species based on pairwise genetic distances and three barcode loci using MEGA 7.0.27 software.

\begin{tabular}{ccccccc}
\hline Gene & Level & No. of Taxa & Min. Dist. (\%) & Mean Dist. (\%) & Max. Dist. (\%) & Se Dist. (\%) \\
\hline \multirow{3}{*}{ ITS2 } & Within Species & 75 & 0.032 & 0.170 & 0.289 & 0.010 \\
& Within Genus & 66 & 0.032 & 0.172 & 0.289 & 0.010 \\
& Within Family & 29 & 0.133 & 0.664 & 0.783 & 0.025 \\
matK & Within Species & 76 & 0.001 & 0.007 & 0.016 & 0.001 \\
& Within Genus & 67 & 0.083 & 0.093 & 0.097 & 0.024 \\
& Within Family & 29 & 0.078 & 0.623 & 0.741 & 0.016 \\
rbcL & Within Species & 77 & 0.000 & 0.005 & 0.030 & 0.001 \\
& Within Genus & 68 & 0.000 & 0.010 & 0.070 & 0.003 \\
& Within Family & 30 & 0.030 & 0.280 & 0.780 & 0.014 \\
\hline
\end{tabular}

Min. dist.: minimum pair wise genetic; Max. dist.: maximum pair wise genetic; Mean dist.: mean pair wise genetic. Se Dist.: Standard error distance.

The outcomes of applying the ABGD algorithm distance values are shown in Figure 2, showing the barcode gap in the three DNA- barcode markers. The ITS2 gene gave a clear gap between the intraspecific (right) and interspecific (left) distances. Analysis was based on K2P genetic distances; the average nucleotide divergence was 0.71-1.05 for the two partitions of distance values obtained from 88 desert plant samples. The pairwise intra-/interspecific genetic distances showed that the matK marker grouped samples into two groups at various genetic distances (60-70): the first was very close to the intraspecific distance, and the second was close to the interspecific distance (0.70-1.0; Figure 2). In the $\mathrm{rbcL}$ marker, the distances were narrow (0.70-0.80) compared to the matK and ITS2 markers.
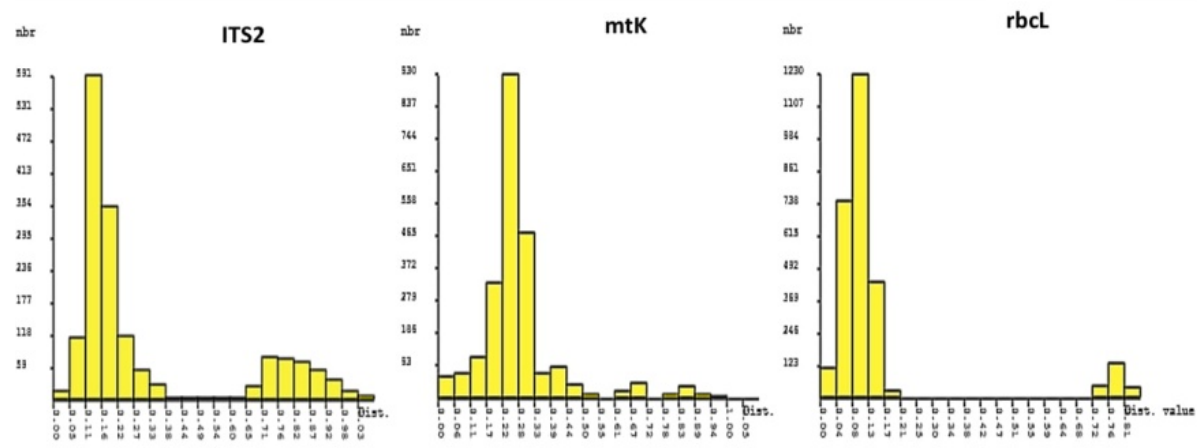

Figure 2. Barcode gaps for three DNA barcodes (ITS2, matK and rbcL) based on the ABGD method and k2p distance between samples of dryland plants. Total barcoded sequences for ITS $2=88$, for matK $=82$ and for rbcL $=90$ individuals.

There were significant variations in the shape of the secondary structure of ITS2 in terms of stem loop number, size and position, which gave unique features to distinguish between the specimens based on the molecular morphological characteristics of these sequences. Figure 3 provides examples of the secondary structure of the ITS2 for some study samples at different taxa levels. 


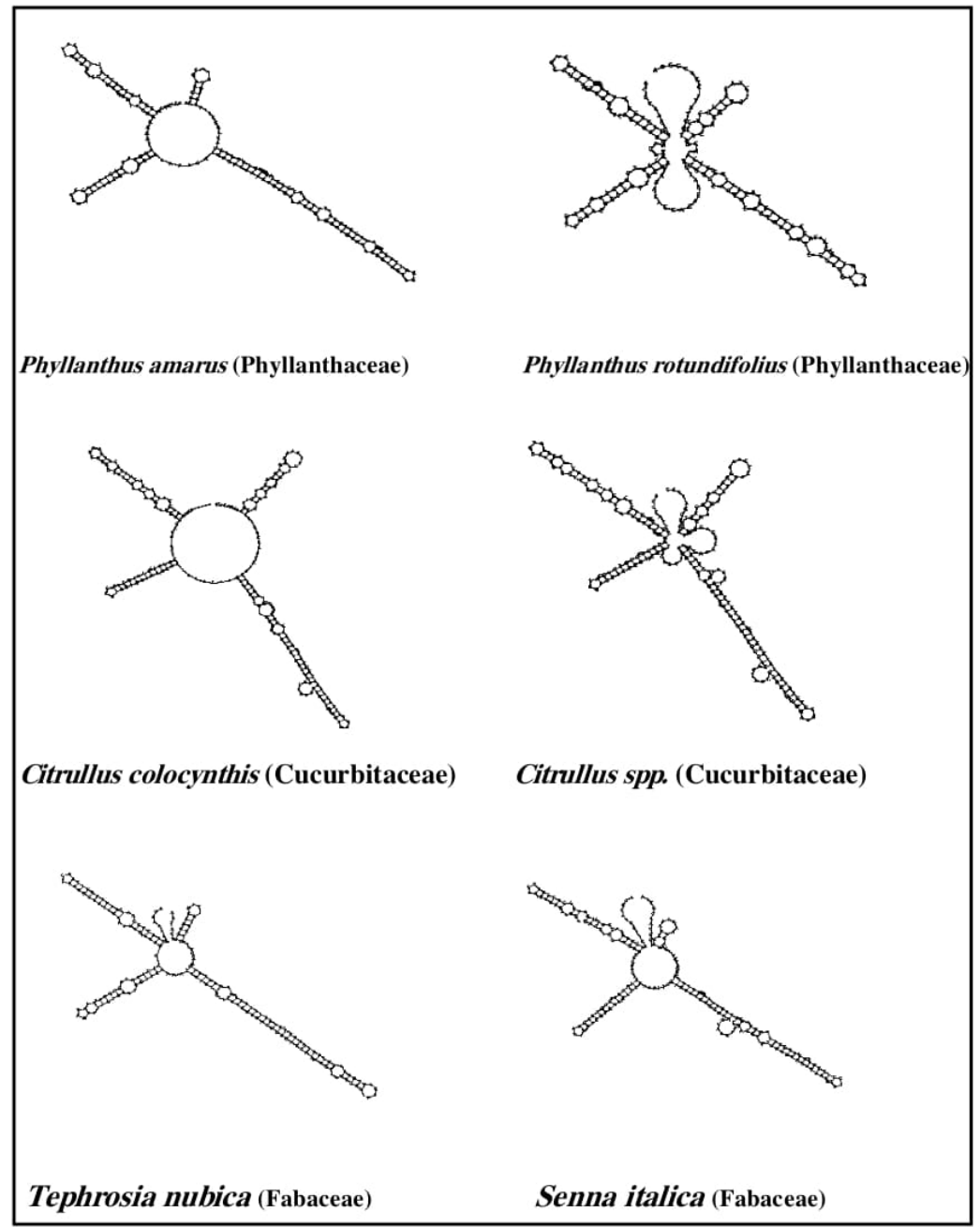

Figure 3. Observed variation in the secondary structure of ITS2 barcodes for six dryland plants under study, and of different taxa levels, using an ITS2 workbench.

\section{Phylogenetic Trees}

The phylogenetic trees (Figures 4, 5 and 6) were based on the NJ method. The NJ tree for ITS2 (Figure 4) was generated from 88 sequences, representing nearly all samples in our study, excluding the two samples that yielded a mixed sequence. Samples of each family were clustered on the same branch of the tree, and each genus was split into sub-branches. For example, Poaceae, Fabaceae, Amaranthaceae, Zygophyllaceae, Apocynaceae, Asteraceae and Brassicaceae were grouped into a clade, the largest cluster being for the first four of these families. There was a high support node $(\geq 60 \%)$ for most samples at the species level within each family. 


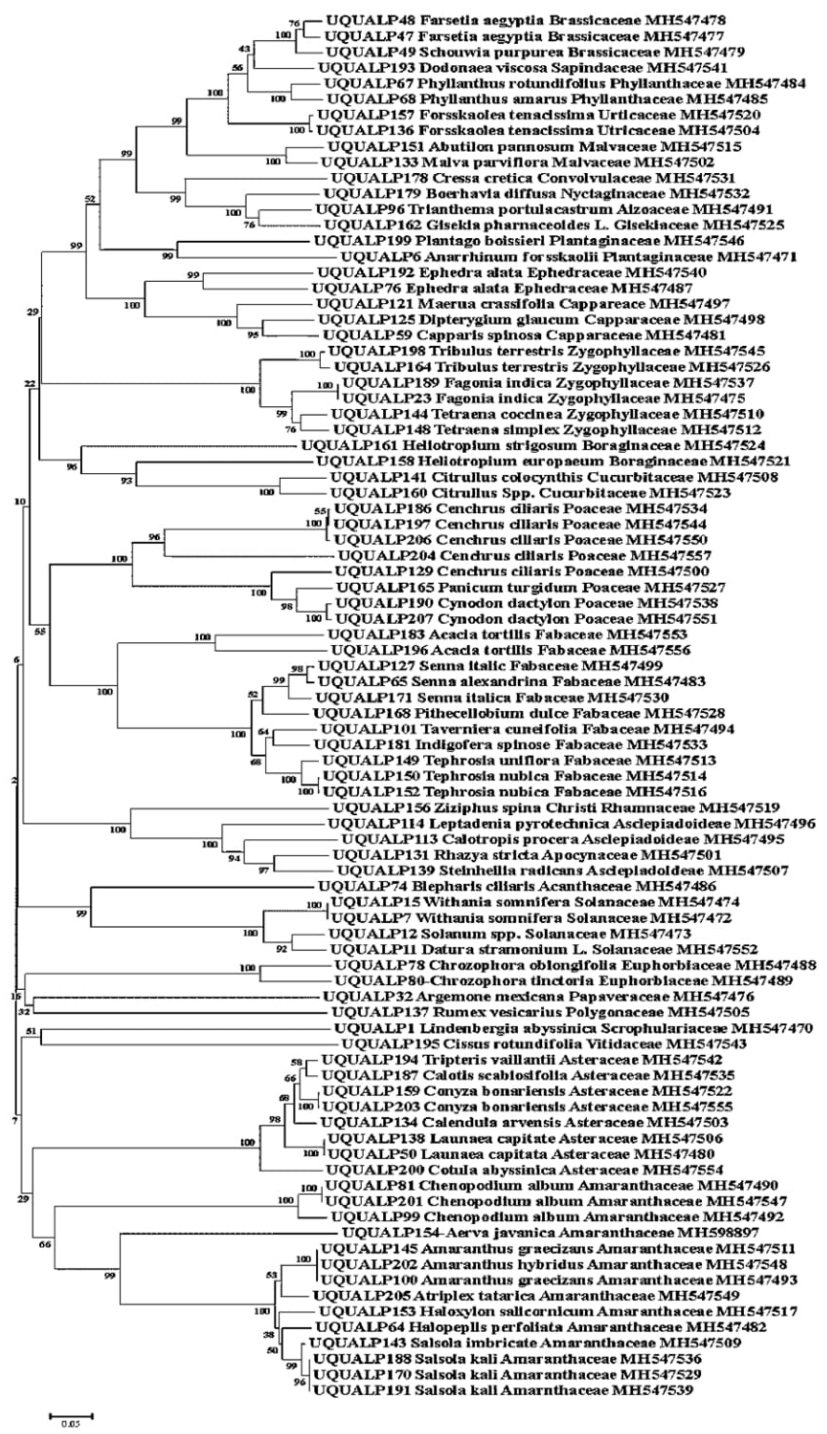

Figure 4. Neighbor joining tree for ITS2 barcodes of 88 dryland plants. Bootstrap replications = 1000; most branches showed strong support $(\geq 60 \%)$ in addition to the sequence accession numbers for each sample.

The NJ tree for the matK gene had 82 sequences (Figure 5). Most species of the same family belonged to one cluster, with strong support for the branches; however, some species of the Asclepiadaceae and Boraginaceae families were not represented in monophyletic groups. In addition, species belonging to the families Euphorbiaceae and Plantaginaceae were clustered in different branches in the rbcL tree, which was constructed of 90 sequences (Figure 6). The ITS2 tree appeared to be adequate in terms of the number of species clustered in the monophyletic group of the right family and the values of the support node. 


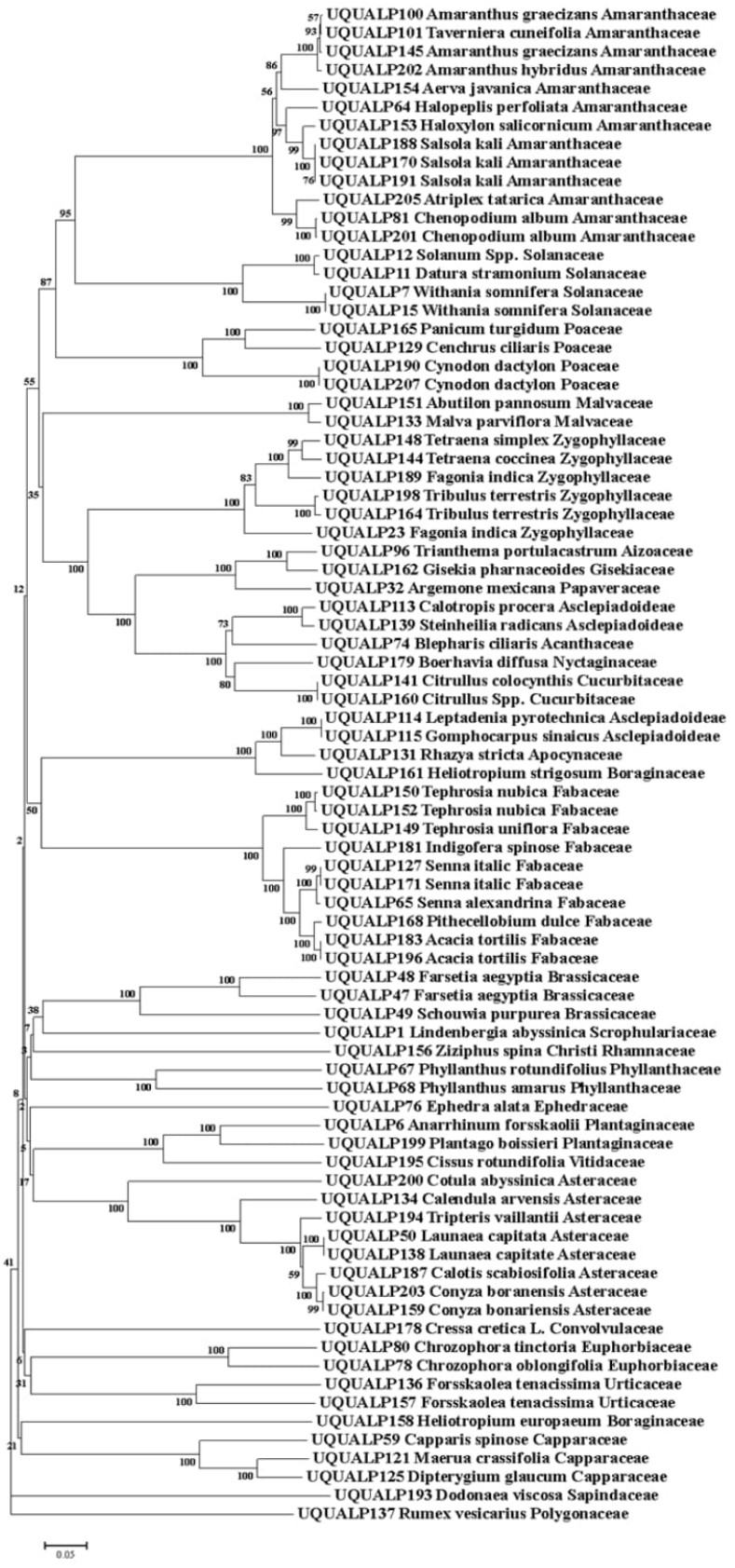

Figure 5. Neighbor joining tree for matK barcodes of 82 dryland plants. Bootstrap replications $=1000$; most branches showed strong support $(\geq 60 \%)$ in addition to the sequence accession numbers for each sample. 


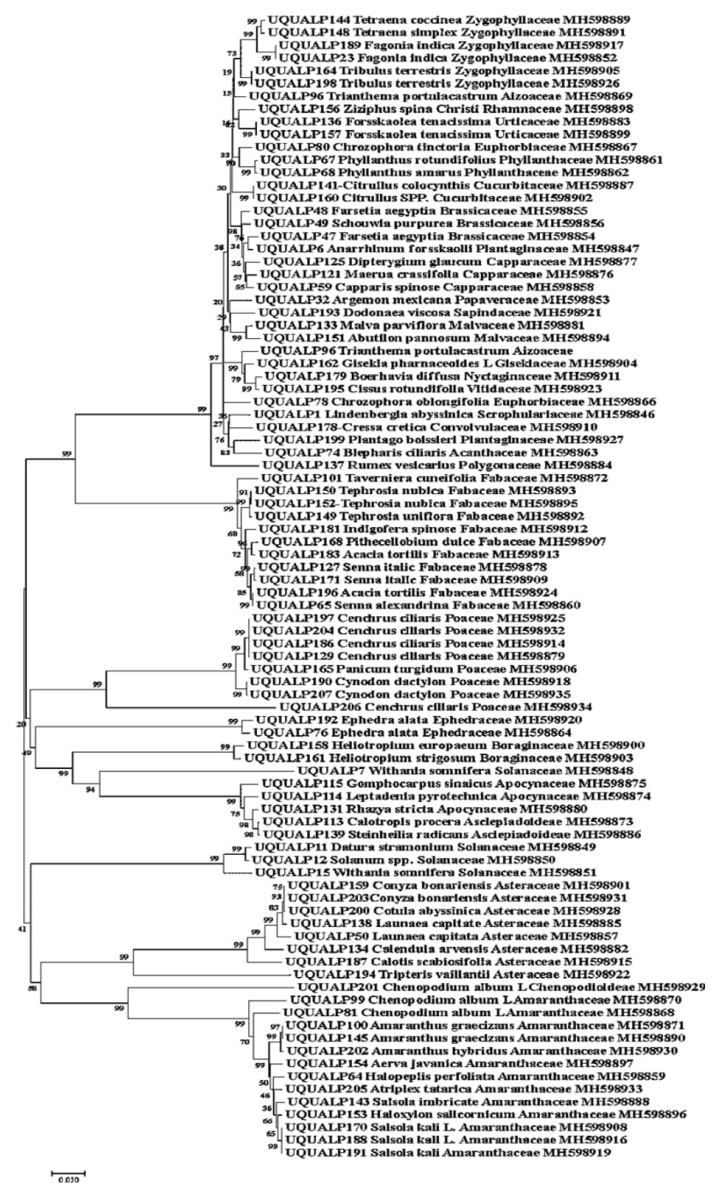

Figure 6. Neighbor joining tree for rbcL barcodes of 90 desert plants. Bootstrap replications $=1000$; most branches showed strong support $(\geq 60 \%)$ in addition to the sequence accession numbers for each sample.

\section{DISCUSSION}

We used three barcode DNA regions (ITS2, matK and rbcL) for identifying and differentiating among 93 specimens of desert plants. Based on the results, the matK region gave difficulties in PCR amplification in several samples and in sequencing, with $88 \%$ efficiency. Meanwhile, the rbcL region gave higher PCR amplification (97\%) and sequencing efficiency (97\%); however the identification efficiencies were low at both the genus $(63.4 \%)$ and species $(45.52 \%)$ levels. This finding was contrary to previous studies that suggested using the rbcL barcode for the identification of rare desert plants. For example, Maloukh et al. (2017) used rbcL and matK loci for a group of 51 native plants (belonging to 22 families) from the United Arab Emirates. Our results are consistent with those of Guo et al. (2016), as these authors mentioned the failure of the chloroplast genes $\mathrm{rbcL}$ and matK in plant specimens identification. This issue is related to the process of inheritance in the plastid region of the plant. Some researchers reported that they were unable to discriminate between many species successfully when they combined the use of 
rbcL and matK (Chen et al., 2010; Michel et al., 2016; Elansary et al., 2017). Chen et al. (2010) mentioned that by using a combination of rbcL and matK, they were able to identify only 550 out of 907 samples (72\%).

In our investigation, the use of the nuclear DNA region ITS2 was the most effective compared with the counterpart chloroplast genes matK and rbcL. ITS2 showed the greatest ability to identify samples at the levels of genus and species (77.2 and 70.4\%, respectively).

Zhang et al. (2015) argue that the most effective DNA barcode region for identification in plants must have a high interspecific genetic divergence and a low intraspecific distance. We found that interspecific divergence was highest in ITS2 $(0.76 \%)$, followed by matK $(0.63 \%)$ and $\mathrm{rbcL}(0.38 \%)$. Another important finding was that the intraspecific distance was lower in ITS2 (0.03) than both rbcL $(0.08)$ and matK $(0.10)$ among the samples studied. This shows the superiority of the ITS2 marker and its relevance for species identification.

The barcode gap is one of the most important tools for evaluating barcode quality (Candek and Kuntner, 2015). In our study, the results of the ABGD algorithm showed a significant barcode gap in ITS2, with clear partitions of distance values (0.71-1.05). The discrimination power of a barcoding region is considered effective if the minimum interspecies distance is greater than its maximum intraspecies distance (Tahir et al., 2018).

The mean genetic distance of the ITS2 locus was significantly greater than the mean distance of both matK and $\mathrm{rbcL}$ loci. This demonstrates the higher resolution power of ITS2. Similar results were reported by Li et al. (2016), who evaluated five DNA barcoding regions (rbcL, matK, trnH-psbA, ITS and ITS2) of 127 individuals representing 40 species and one variety of Oberonia from China. All three plastid candidates tested (rbcL, matK and trnH-psbA) had a lower discriminatory power than the nuclear regions (ITS and ITS2), and the ITS2 region had the highest resolution rate (82.14\%). This study supports what has been mentioned previously by (Hollingsworth, 2011), who referred to possibility to used ITS2 region as alternatives to amplify ITS region in plant and it has a high discrimination power, where it is amplified part of ITS region, this will be useful if it difficult to amplify the whole nrDNA ITS region. This again supports the use of the nuclear gene ITS2 for the identification of local flora and for phylogeny studies.

Our findings confirm the effectiveness of the ITS2 region and support the proposal that the ITS2 region is universal in plants as a DNA barcode, corresponding to the CO1 region in animals (Chen et al., 2010). If this is the case, ITS2 has great potential for identifying plants at both higher and lower levels. The China Plant BOL Group has mentioned that The advantages of using nuclear DNA barcode ITS was outweigh its disadvantages, and it will contribute to solving problems related to identification at the species level (Hollingsworth, 2011). Braukmann et al. (2017) pointed out an important property that supports the use of the ITS2 gene for standard DNA barcoding in plants: its short length. This property makes amplification possible with fewer primers. The superiority of nuclear genes could be explained by the abundant quantity of genetic information and gene flow available in nuclear regions ( Petit and Excoffier, 2009; Naciri et al., 2012).

In our study, we tested ITS2 on 93 samples grown in a harsh climate with difficult environmental conditions. Many were preserved as old herbarium specimens, some of which were in poor conservation conditions and showed evidence of degradation. Our results show that ITS2 had a 97\% amplification capacity with 77.2 and $70.4 \%$ successful 
identification at the genus and species levels, respectively. Our results agree with those of Yu et al. (2017), who found that the ITS2 region was able to detect the identity of degraded specimens because ITS2 is a multi-copy region.

Recent studies have recommended the use of ITS2 to verify the content of traditional medicine material. Han et al. (2016) examined 1,436 specimens of 295 medicinal species in China. The results confirmed that ITS2 barcodes could be amplified and sequenced for most of the samples $(87.7 \%)$. The ITS2 barcode was successfully used to identify 58 herbal medicinal plants, including samples of M. tenacissima and its adulterants (Yu et al., 2018). As well as a way to reveal commercial fraud in the herbal industry, especially if products are falsely sold as powdered plant material in traditional shops (Michel et al., 2016), it can also be used as a reliable means to verify the identity of herbs used in the pharmaceutical industry, in addition to traditional morphological and chemical methods, DNA barcoding with ITS2 has recently been used as a standard quality control procedure in several studies (Khan et al., 2017; Yu et al., 2017)

In some cases, ITS2 failed in species identification in our study. A possible explanation for this might be that the sequences stored in GeneBank were misidentified. This was also reported by Griffiths et al. (2006), Michel et al. (2016) and Elansary et al. (2017).

Our study shows sequences of a group of species that have not previously been available in the NCBI and Barcode of Life databases. Therefore, the sequences presented in this study, and those expected to result from future studies, will help to resolve the lack of ITS2 sequences.

\section{CONCLUSIONS}

In recent years, the ITS2 region has been commonly used in plant identification, taxonomy and molecular phylogeny for the verification of medicinal plants. In our study, the DNA barcode ITS2 was capable of pinpointing the taxonomic identity of most species tested. This is because ITS2 had a higher barcoding success rate and species resolution when compared to matK and rbcL markers. We recommend the use of ITS2 as a standard barcode for identifying desert plants, increasing its testing in future studies with a larger number of dryland samples.

According to our results, ITS2 is successful not only in amplifying and sequencing but also in identification, and it could, therefore, be applied in cases of old herbal specimens, even with damaged or old samples. This will further enhance conservation efforts at the local and global levels.

At the local level, there is a need to apply barcode markers to a wide range of local flora for the purposes of identification, taxonomy and phylogeny. More broadly, further efforts are needed to ensure the conservation of desert plants, especially those of medical importance; using barcode technology will enhance these efforts.

\section{FUNDING}

This research did not receive any specific grant from funding agencies in public, commercial or not-for-profit sectors. 


\section{ACKNOWLEDGMENTS}

I would like to extend my thanks to Umm Al-Qura University for allowing me to access its herbarium and for allowing me to make use of its facilities.

\section{REFERENCES}

Abdel KK, El-Shikh M and El-Aidarous A (2013). Floristic diversity and vegetation of Wadi al-Noman, Mecca, Saudi Arabia. Turkish J. Bot. 37: 894-907.

Alaklabi A, Arif IA, Bafeel SO, Alfarhan AH, et al. (2014). Nucleotide based validation of the endangered plant Diospyros mespiliformis (Ebenaceae) by evaluating short sequence region of plastid rbcL gene. Plant Omics 7: 102-107.

AL-Eisawi DMA and Al-Ruzayza S (2015). The flora holy Mecca district, SaudiArabia. IJBC 7: 173-189.

Ali MA, Al-Hemaid FM, Lee J, Hatamleh AA, et al. (2015). Unraveling systematic inventory of Echinops (Asteraceae) with special reference to nrDNA ITS sequence-based molecular typing of Echinops abuzinadianus. Genet. Mol. Res. 14: 11752-11762.

Al-Qurainy F, Khan S, Nadeem M, Tarroum M, et al. (2013). Assessment of phylogenetic relationship of rare plant species collected from Saudi Arabia using internal transcribed spacer sequences of nuclear ribosomal DNA. Genet. Mol. Res. 12: 723-730.

Al-Qurainy F, Khan S, Nadeem M, Tarroum M, et al. (2014). Selection of DNA barcoding loci for Nepeta deflersiana Schweinf. ex Hedge from chloroplast and nuclear DNA genomes. Genet. Mol. Res. 13: 1144-1151.

Arolla RG, Cherukupalli N, Khareedu VR and Vudem DR (2015). DNA barcoding and haplotyping in different species of Andrographis. Biochem. Syst. Ecol. 62: 91-97.

Azimi S, Nixon G, Ahern J and Balachandran W (2011). A magnetic bead-based DNA extraction and purification microfluidic device. Microfluid Nanofluidics 11: 157-165

Bafeel SO, Alaklabi A, Arif IA, Khan H, et al. (2012a). Molecular characterization of regionally endangered tree species Mimusops laurifolia (Forssk.) Friis (Sapotaceae). Int. J. Biol. 4: 29-37.

Bafeel SO, Alaklabi A, Arif IA, Khan H, et al. (2012b). Ribulose-1, 5-biphosphate carboxylase (rbcL) gene sequence and random amplification of polymorphic DNA (RAPD) profile of regionally endangered tree species Coptosperma graveolens subsp. arabicum (S. Moore). Plant Omics 5: 285-290.

Bafeel SO, Arif IA, Al-Homaidan AA, Khan H, et al. (2012c). Assessment of DNA barcoding for the identification of Chenopodium murale L. (Chenopodiaceae). Int. J. Biol. 4: 66-74.

Bafeel SO, Arif IA, Bakir MA, Khan H, et al. (2011). Comparative evaluation of PCR success with universal primers of maturase $\mathrm{K}$ (matK) and ribulose-1, 5-bisphosphate carboxylase oxygenase large subunit (rbcL) for barcoding of some arid plants. Plant Omics 4: 195-198.

Braukmann TW, Kuzmina ML, Sills J, Zakharov E, et al. (2017). Testing the efficacy of DNA barcodes for identifying the vascular plants of Canada. PLoS ONE 12: e0169515.

Candek K and Kuntner M (2015). DNA barcoding gap: Reliable species identification over morphological and geographical scales. Mol. Ecol. Resour. 15: 268-277.

CBOL Plant Working Group (2009). A DNA barcode for land plants. Proc. Natl. Acad. Sci. U.S.A. 106: 12794-12797.

Chaudhary SA (2000). Flora of the Kingdom of Saudi Arabia Illustrated. Ministry of Agriculture and Water: National Agriculture Research Center, Riyadh, Saudi Arabia.

Chaudhary SA (2001). Flora of the Kingdom of Saudi Arabia Illustrated. Ministry of Agriculture and Water: National Agriculture Research Center, Riyadh, Saudi Arabia.

Chen S, Yao H, Han J, Liu C, et al. (2010). Validation of the ITS2 region as a novel DNA barcode for identifying medicinal plant species. PLOS ONE 5: e8613.

Elansary OH, Ashfaq M, Ali HM and Yessoufou K (2017). The first initiative of DNA barcoding of ornamental plants from Egypt and potential applications in horticulture industry. PLOS ONE 12: e0172170.

Fazekas A, Burgess K, Kesanakurti P, Graham S, et al. (2008). Multiple multilocus DNA barcodes from the plastid genome discriminate plant species equally well. PLoS ONE 3: e2802. Available at [https://doi.org/10.1371/journal.pone.0002802(22/6/2018)].

Gao T, Yao H, Song J, Liu C, et al. (2011). Identification of medicinal plants in the family Fabaceae using a potential DNA barcode ITS2. J. Ethnopharmacol. 130: 116-121.

Griffiths A, Miller J, Suzuki D, Lewontin R, et al. (2006). An introduction to genetic analysis. W. H. Freeman and Company, New York.

Guo Y-Y, Huang L-Q, Liu Z-J and Wang X-Q (2016). Promise and challenge of DNA barcoding in Venus Slipper (Paphiopedilum). PLoS ONE 11: $\mathrm{e} 0146880$. 
Han J, Pang X, Liao B, Hui Y, et al. (2016). An authenticity survey of herbal medicines from markets in China using DNA barcoding. Sci. Rep. 6: 1-9.

Hebert PD, Cywinska A, Ball SL and deWaard JR (2003). Biological identifications through DNA barcodes. Proceeding Biological Science 270: 313-321.

Hollingsworth PM (2011). Refining the DNA barcode for land plants. PNAS. 108(49): 19451-19452.

Keller A, Schleicher T, Schultz J, Muller T, et al. (2009). 5.8S-28S rRNA interaction and HMM-based ITS2 annotation. Gene 430: 50-57.

Khan S, Al-Qurainy F, Nadeem M and Tarroum M (2012a). Development of genetic markers for Ochradenus arabicus (Resedaceae), an endemic medicinal plant of Saudi Arabia. Genet. Mol. Res. 11: 1300-1308.

Khan SA, Baeshen MN, Ramadan HA and Baeshen NA (2017). Emergence of plastidial intergenic spacers as suitable DNA barcodes for arid medicinal plant Rhazya stricta. Am J Plant Sci 8: 1774-1789.

Kumar S, Stecher G and Tamura K (2016). MEGA7: Molecular Evolutionary Genetics Analysis Version 7.0 for Bigger Datasets. Mol. Biol. Evol. 33: 7. 1870-1874. https://doi.org/10.1093/molbev/msw054.

Li Y, Tong Y and Xing F (2016). DNA Barcoding Evaluation and ITS Taxonomic Implications in the Recently Evolved Genus Oberonia Lindl. (Orchidaceae) in China. Front Plant Sci. 7: 1791.

Lin J-Y, Lin B-Y, Chang C-D, Liao S-C, et al. (2015). Evaluation of chloroplast DNA markers for distinguishing Phalaenopsis species. Sci. Hortic. 192: 302-310.

Maloukh L, Kumarappan A, Jarrar M, Salehi J, et al. (2017). Discriminatory power of rbcL barcode locus for authentication of some of United Arab Emirates (UAE) native plants. 3 Biotech 7: 144.

Martin MP, Finschow G, Salom JC, Siquier JLl, et al. (2016). Barcoding sequences clearly separate Chroogomphus mediterraneus (Gomphidiaceae, Boletales) from C. rutilus, and allied species. Mycoscience 57: 384-392.

Meelad MMHS (1987). Some floristic and chemotaxonomic studies of the flowering plants of Makkah. Doctoral dissertation. The University of Salford, Salford, Manchester, UK.

Meier R, Kwong S, Vaidya G and Ng PKL (2006). DNA barcoding and taxonomy in Diptera: A tale of high intraspecific variability and low identification success. Syst. Biol. 55: 715-728.

Mezzasalma V, Bruni I, Fontana D, Galimberti A, et al. (2017). A DNA barcoding approach for identifying species in Amazonian traditional medicine: The case of Piri-Piri. Plant Gene 9: 1-5.

Michel C-I, Meyer RS, Taveras Y and Molina J (2016). The nuclear internal transcribed spacer (ITS2) as a practical plant DNA barcode for herbal medicines. J Appl Res Med Aromat Plants 3: 94-100.

Migahid AM (1996). Flora of Saudi Arabia. Riyadh University, Riyadh, Saudi Arabia.

Mohamed A (2016). DNA Barcoding of Five Medicinal Plants from Siwa Oasis, Egypt. KMITL Sci. Tech. 16: 49-56.

Naciri Y, Caetano S and Salamin N (2012). Plant DNA barcodes and the influence of gene flow. Mol. Ecol. Resour. 12: 575-580.

Nithaniyal S and Parani M (2016). Evaluation of chloroplast and nuclear DNA barcodes for species identification in Terminalia L. Biochem. Syst. Ecol. 68: 223-229.

Pan Y-Z, Fang L-Q, Hao G and Gong X (2009). Systematic positions of Lamiophlomis and Paraphlomis (Lamiaceae) based on nuclear and chloroplast sequences. Plant Syst. Evol. 47: 535-542.

Patil SM, Rane NR, Adsul AA, Gholave AR, et al. (2016). Study of molecular genetic diversity and evolutionary history of medicinally important endangered genus Chlorophytum using DNA barcodes. Biochem. Syst. Ecol. 65: 245-252.

Petit RJ and Excoffier L. (2009). Gene flow and species delimitation. Trends Ecol. Evol. (Amst.) 24: 386-393.

Puillandre N, Lambert N, Brouillet S and Achaz G (2012). ABGD, automatic barcode gap discovery for primary species delimitation. Mol. Ecol. 21: 1864-1877.

Sarvananda L (2018). Short introduction of DNA barcoding. IJR. 5: 673-686.

Scriver M, Marinich A, Wilson C and Freeland J. (2015). Development of species-specific environmental DNA (eDNA) markers for invasive aquatic plants. Aquat. Bot. 122: 27-31.

Schultz J, Maisel S, Gerlach D and Muller T (2005). A common core of secondary structure of the internal transcribed spacer 2 (ITS2) throughout the Eukaryota. RNA 11: 361-364. Available at [http://its2.bioapps.biozentrum.uniwuerzburg.de/].

Sher H. and Aldosari AA (2012). Overview on the ecological and geographical appraisal of important medicinal and aromatic plants: An endangered component in the flora of Saudi Arabia. Sci. Res. Essays 7: 1639-1646.

Silva JN, Bezerra da Costa A, Silva JV and Almeida C (2015). DNA barcoding and phylogeny in neotropical species of the genus Spondias. Biochem. Syst. Ecol. 61: 240-243.

Tahir A, Hussain F, Ahmed N, Ghorbani A, et al. (2018). Assessing universality of DNA barcoding in geographically isolated selected desert medicinal species of Fabaceae and Poaceae. Peer J. 4499: 1-16.

Tamura K, Peterson D, Peterson N, Stecher G, et al. (2011). MEGA5: Molecular evolutionary genetics analysis using maximum likelihood, evolutionary distance, and maximum parsimony methods. Mol. Biol. Evol. 28: 2731-2739.

Vincent P (2008). Saudi Arabia: An Environmental Overview. London: Taylor \& Francis.

Xiwen L, Yang Y, Robert JH, Maurizio R, et al. (2015). Plant DNA barcoding: From gene to genome. Biol. Rev. 90: 157-166.

Yang T, Zhang T, Guo Y-h and Liu X (2017). Testing eight barcoding markers for Potamogeton species at intraspecific levels. Aquat. Bot. 137: 56-64.

Genetics and Molecular Research 18 (1): gmr18133 
Yu N, Wei Y, Zhang X, Zhu N, et al. (2017). Barcode ITS2: A useful tool for identifying Trachelospermum jasminoides and a good monitor for medicine market. Scientific Reports 7: 5037.

Yu N, Wei Y, Zhu Y, Zhu N, et al. (2018). Integrated approach for identifying and evaluating the quality of Marsdenia tenacissima in the medicine market. PLOS ONE 13: e0195240.

Zhang Z-L, Song M-F, Guan Y-H, Li H-T, et al. (2015). DNA barcoding in medicinal plants: Testing the potential of a proposed barcoding marker for identification of Uncaria species from China. Biochem. Syst. Ecol. 60: 6-14n. Bot. 59: 18 - 28 